\title{
Analyse Spatiale de l'évolution du Trait de Côte Autour de l'embouchure du Fleuve Casamance (Sénégal) de 1968 à 2017, à Partir de l'outil DSAS
}

\section{Mamadou Thior, \\ Tidiane Sane,}

Laboratoire de Géomatique et d'Environnement (LGE),

Université Assane SECK de Ziguinchor, Ziguinchor, Sénégal,

Laboratoire Mixte International «Patrimoines et Territoires de l'Eau »

(LMI PATEO), Institut de Recherche pour le Développement (IRD)

\section{Oumar Sy,}

Laboratoire de Géomatique et d'Environnement (LGE),

Université Assane SECK de Ziguinchor, Ziguinchor, Sénégal

\section{Luc Descroix,}

Laboratoire Mixte International «Patrimoines et Territoires de l'Eau »

(LMI PATEO), Institut de Recherche pour le Développement (IRD)

\section{Boubacar Demba Ba, Boubacar Solly, Victor Mendy,}

Laboratoire de Géomatique et d'Environnement (LGE),

Université Assane SECK de Ziguinchor, Ziguinchor, Sénégal, Laboratoire Mixte International «Patrimoines et Territoires de l'Eau » (LMI PATEO), Institut de Recherche pour le Développement (IRD)

\section{Résumé}

Située au sud-ouest du littoral sénégalais, l'embouchure du fleuve Casamance est sous l'influence des facteurs hydrodynamiques naturels. Ainsi, les plages des villages situés au tour de cette embouchure sont caractérisées par une mobilité très variable. L'objectif de cet article est d'analyser la variation de la position du trait de côte autour de l'embouchure du fleuve Casamance. La méthodologie est basée sur la superposition d'une série d'images multi-dates (photographies aériennes, images Landsat et image Google Earth). Les travaux de numérisation ont été faits avec le logiciel ArcGIS 10.5. A partir de là, les calculs statistiques des vitesses d'évolution du trait de côte sont effectués avec le modèle de DSAS4.3. Les résultats obtenus à travers le calcul des indices de mobilité (EPR et LRR) ont montré 
une dynamique très variable du trait de côte dégageant deux tendances : une première période, comprise entre 1968 et 1986, caractérisée globalement par une progradation et une seconde période (1986-2017) caractérisée globalement par un recul de la plage, mais avec un recul moins rapide entre 2004 et 2017.

Mots clés: Analyse spatiale, Trait de côte, modèle DSAS, Embouchure, Fleuve Casamance

\section{Analyse Spatiale de l'évolution du Trait de Côte Autour de l'embouchure du Fleuve Casamance (Sénégal) de 1968 à 2017, à Partir de l'outil DSAS}

\section{Mamadou Thior, Tidiane Sane,}

Laboratoire de Géomatique et d'Environnement (LGE), Université Assane SECK de Ziguinchor, Ziguinchor, Sénégal, Laboratoire Mixte International «Patrimoines et Territoires de l'Eau » (LMI PATEO), Institut de Recherche pour le Développement (IRD)

\section{Oumar Sy,}

Laboratoire de Géomatique et d'Environnement (LGE),

Université Assane SECK de Ziguinchor, Ziguinchor, Sénégal

\section{Luc Descroix,}

Laboratoire Mixte International «Patrimoines et Territoires de l'Eau » (LMI PATEO), Institut de Recherche pour le Développement (IRD)

\section{Boubacar Demba Ba, Boubacar Solly, Victor Mendy,}

Laboratoire de Géomatique et d'Environnement (LGE),

Université Assane SECK de Ziguinchor, Ziguinchor, Sénégal, Laboratoire Mixte International «Patrimoines et Territoires de l'Eau » (LMI PATEO), Institut de Recherche pour le Développement (IRD)

\section{Abstract}

Located south-west of the Senegalese coast, the mouth of the Casamance River is under the influence of natural hydrodynamic factors. Thus, the beaches of the villages located around this mouth are characterized 
by a highly variable mobility. The purpose of this article is to analyze the variation of the position of the coastline around the mouth of the Casamance River. The methodology is based on the superimposition of a series of multidate images (aerial photographs, Landsat images and Google Earth image). Scanning jobs were done with ArcGIS 10.5 software. From there, the statistical calculations of the rates of evolution of the coastline are made with the model of DSAS4.3. The results obtained through the calculation of the mobility indices (EPR and LRR) showed a very variable dynamics of the coastline revealing two trends: a first period between 1968 and 1986, characterized generally by a progradation and a second period (1986- 2017) characterized in general by a decline in the beach, but with a slower decline between 2004 and 2017.

Keywords: Spatial Analysis, Coastline, DSAS model, Mouth, Casamance River

\section{Introduction}

L'analyse de l'évolution du trait de côte (cinématique) du littoral de la Casamance en général et de l'embouchure en particulier, exige le choix d'un indicateur. En effet, des études réalisées sur la question ont montré qu'il existe plus d'une douzaine de lignes de références matérialisant la position du trait de côte (Himmelstoss, 2009 ; Thieler, et al., 2003 ; Fletcher et al., 2003 ; Boak et al., 2005) Il s'agit donc de mettre au point une méthode d'extraction et de suivi du marqueur utilisé selon le type de côte, le matériel et les données disponibles. Il existe en effet une diversité de méthodes pour détecter, extraire, et suivre la dynamique du trait de côte (Moore 2000 ; Le Berre, 2016). Mais, les différentes approches méthodologiques reposent sur la compilation et la comparaison de données acquises, soit sur le terrain par des instruments de topométrie (niveau de chantier, théodolite, tachéomètre électronique, lidar, récepteur DGPS, etc.), soit en laboratoire par le traitement numérique d'images satellites ou aériennes (Faye, 2010, Emmanuel, 2017). Ainsi, dans cette étude, il s'agite de mettre en œuvre le traitement numérique pour analyser la mobilité du trait de côte. L'étude du linéaire côtier nécessitant un choix d'une ligne de référence, la limite de la végétation a été utilisée comme pour représenter de la position du trait de côte. Ce choix est lié au fait que la végétation est non seulement bien présente sur la côte de la Casamance, mais aussi, elle est visible sur les données géospatiales. Le village de Diogué au nord, l'île de Carabane et la pointe de Gnikine au sud ont servi de site de suivi (fig. 1). 


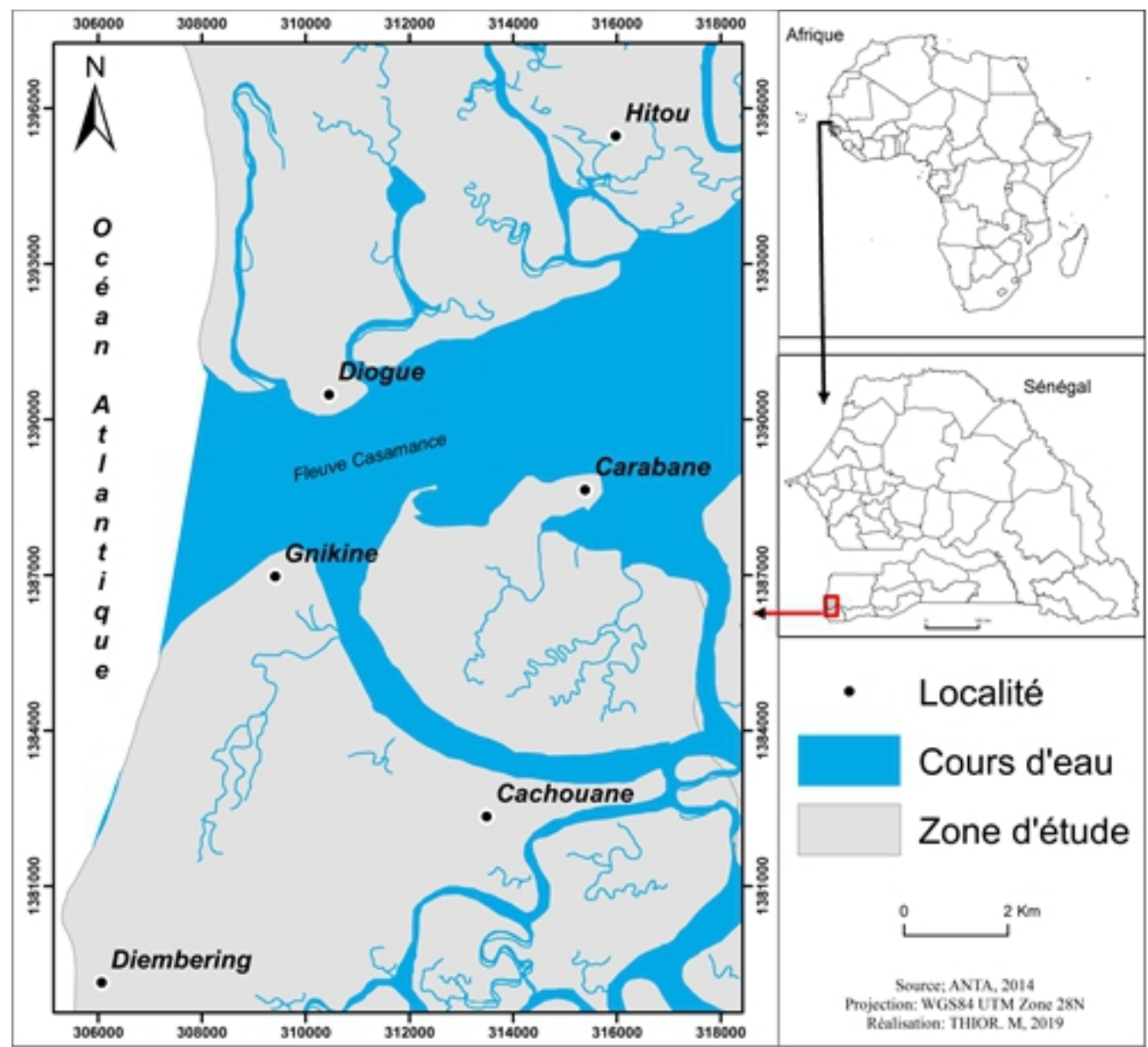

Figure 1: Localisation des sites d'étude de l'embouchure du fleuve Casamance

\section{Matériels et Méthodes}

L'extraction des repères s'est faite à partir de photographies aériennes, d'images satellitaires et de documents cartographiques anciens. Ces données sont considérées comme étant les principales données pour analyser l'évolution historique des littoraux. Toutefois, elles présentent des sources d'erreurs (Trébossen et al., 2005). Dans cette étude, nous avons utilisé ces différents types de données géospatiales sur quatre dates. Il s'agit d'une photographie aérienne de 1968, d'une image satellitaire Landsat de 1986, et des images Google Earth Pro de 2004 et 2017.

La résolution spatiale de la photographie aérienne de 1968 (1 m) offre la possibilité de voir la limite de la végétation.

Sur les images Landsat 1986, la limite de la végétation en front de plage, qu'elle soit dunaire (Vegetation line, seaward edge of dune vegetation) ou pérenne (Line of permanent, stable, long-term vegetation), est une entité bien visible si une composition colorée correspondante y est appliquée. 
Les premiers traitements par télédétection des images satellitaires de 1986 que nous avons utilisées ont été faits avec le logiciel ENVI 4.5. Du visible à l'infrarouge, plusieurs bandes sont fournies par le capteur TM. L'approche a été d'accorder plus d'importance aux informations ciblées sur le terrain dans le choix des bandes spectrales étant donné que la végétation dans ce choix est incontournable pour l'extraction de sa limite. Il fait mieux ressortir le contact entre les sédiments secs de la haute plage et la couverture végétale (Robin et al., 2016; Le Berre et al., 2016). Ensuite un seuillage a permis d'identifier grâce à une limite nette (ligne de référence) les populations de pixels représentant la zone terrestre (pixels clairs) et le domaine marin (pixels sombres) (Djagouna., 2016).

Sur les images de Google Earth dont les plus anciennes datent de 2004, la résolution spatiale varie en fonction du niveau de zoom. En effet, avec une résolution de $-1 \mathrm{~m}$, il est facile dans certains endroits de détecter presque tous les types de ligne de référence. Par ailleurs, plus la zone à télécharger est petite plus sa qualité est bonne. Ce qui fait que sur une zone ciblée, nous avons réalisé plusieurs petites captures, puis elles sont mosaïquées sur l'interface cartographique d'ArcGIS. À la suite de la mosaïque, l'image est ensuite géoréférencée pour pouvoir être projetée dans un système de coordonnées WGS84 UTM Zone 28N.

\subsection{Les marges d'erreurs}

Dans la littérature, l'erreur globale appelée Erreur de Position Globale du Trait de côte est calculée en prenant la racine carrée de la somme des carrées de chaque erreur (Mathieu 2014 ; Juigner 2012 ; Fletcher et al., 2003). Ainsi, dans cette étude, on calcule pour chaque année la valeur des trois erreurs déjà identifiées sur les images. Cela donne donc la formule suivante pour chaque date :

$$
\begin{gathered}
\boldsymbol{E p t}=\sqrt{E p^{2}+E g^{2}+E d^{2}} \\
\boldsymbol{E} \boldsymbol{\alpha}=\frac{\sqrt{E p t 1^{2}+E p t 2^{2}}}{\text { Période }}
\end{gathered}
$$

$\mathbf{E}_{\mathbf{p t}}$ : Erreur de position globale du trait de côte

$\mathbf{E}_{\mathbf{p}: \text { Erreur de pixel (résolution spatiale) }}$

$\mathbf{E}_{\mathrm{g}}$ : Erreur de Géoréférencement (RMS)

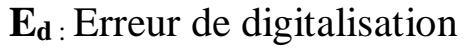

$\mathbf{E} \boldsymbol{\alpha}$ : Erreur globale en moyenne en m/an

La somme de ces racines carrées donne l'erreur d'un pas de temps étudié. Ainsi, pour intégrer la marge d'erreur sur les résultats d'analyse, il faut obtenir le cumul des sommes des erreurs de position globale du trait de côte pour chaque pas de temps d'analyse. 


\subsection{Calculs statistiques sur Digital Shoreline Analysis System (DSAS)}

Les travaux de numérisation ont été faits sur le logiciel ArcGIS 10.5, à partir duquel, les calculs statistiques des vitesses d'évolution du trait de côte sont effectués à l'aide de l'extension DSAS4.3. En effet, DSAS est une extension qui permet de faire des calculs sur les écarts des traits de côte déjà digitalisés à partir des images retenues.

Le calcul de la vitesse d'évolution du trait de côte nécessite préalablement la mise en place d'au moins deux traits de côte numérisés à différentes dates. Ainsi, avec l'outil DSAS couplé avec ArcGis, le calcul des taux d'évolution est automatisé suivant les manipulations exigées. Le principe général de cet outil est de mesurer les écarts entre les traits de côte d'une même série mais aussi de calculer les statistiques des taux d'évolution (en m/an). Pour cela, l'exploitation de l'outil demande au préalable une mise en forme rigoureuse des données dans une géodatabase personnelle, la création d'une ligne de base et de transects équidistants, une estimation de l'incertitude liée à la méthode ainsi que le choix des statistiques de calculs des taux d'évolutions (Juigner, 2012; Crowell et al., 1994). Ainsi, lorsque tous les paramètres d'entrées sont correctement saisis, DSAS génère automatiquement selon le pas de mesure défini des transects. Les transects sont perpendiculaires au linéaire côtier et mesurent les écarts entre les traits de côte puis calculent les taux moyens de déplacement le long de chaque transect (Faye et al., 2010).

\subsection{Les indices calculés}

La représentation des résultats est faite en deux échelles. La première correspond à l'écart entre deux dates (deux traits de côte) et la seconde présentation correspond à l'échelle globale et prend en compte tous les traits de côte (1968, 1986, 2004 et 2017).

L'End Point Rate (EPR) permet de mesurer l'écart entre deux traits de côte successifs. Il s'agit du rapport de la distance entre le trait de côte correspondant à la date la plus ancienne et la plus récente. Ainsi, lorsqu'on dispose uniquement de deux traits de côte, l'EPR reste un bon indice pour évaluer la cinématique (Thieler et al., 2005 ; Moore, 2000). C'est pourquoi nous l'avons utilisé pour évaluer les variations entre 1968-1986, 1986-2004 et 2004-2017.

Le Low Regression Rate-of-change (LRR) lui permet d'estimer l'évolution de chaque segment sur l'ensemble de la période considérée. Le nombre d'individus de la série statistique est celui du nombre de traits de côte. Ainsi, si ce nombre est faible comme pour l'EPR, cela pose le problème de la pertinence et de la robustesse des résultats. Ainsi, calculant le taux d'évolution annuelle de la ligne de référence le long de chaque transect, l'EPR est donc plus pertinent lorsqu'on analyse la cinématique littorale pour plus de deux 
dates. En effet, la méthode de calcul tient compte de la distance évolutive du trait de côte sur toute la période considérée (Faye et al., 2010).

\subsection{Les opérations de pré calculs}

Il s'agit de créer tout au début une Géodatabase personnelle composée essentiellement de deux entités: une première entité contenant une ou plusieurs ligne (s) de références nommées baseline et une seconde entité constituée de traits de côte déjà numérisés sur ArcMap nommée shorelines. Ainsi, la zone tampon Buffer shoreline permet de stabiliser l'espace linéaire dans lequel les traits de côte ont été numérisés.

\section{La Baseline}

Il s'agit de numériser une ligne de base imaginaire à partir de laquelle DSAS crée des transects qui coupent les différents traits de côtes. Ainsi, tous les transects sont perpendiculaires à la baseline qui elle aussi doit être parallèle à la côte. Cependant, il arrive que les transects soient déformés du fait de l'irrégularité de la côte. Ce qui entraîne parfois des transects aberrants ou qui s'entrecoupent avant leur intersection sur les traits de côte. Il est ainsi possible de les corriger en les redressent ou de les supprimer

\section{$>$ Les shorelines}

Les shorelines représentent les traits de côte déjà numérisés dans la Géodatabase. Il s'agit en effet, des traits de côte auxquels la baseline doit être parallèle et devant servir de mesure dans l'environnement de DSAS. Ainsi, pour pouvoir effectuer un calcul d'indices (EPR, LRR) les shorelines doivent être au minimum de deux entités de dates ou encore d'heures différentes.

\section{Les Buffer shoreline}

Pour effectuer un calcul sur DSAS, il faut circonscrire le segment sur lequel les mesures de variation de l'évolution du trait de côte seront réalisées. C'est en effet la zone tampon qui permet de définir de quel côté de la baseline seront tracés les profils. En effet, la zone tampon donne deux possibilités de position de la baseline (du côté de la mer ou du côté de la terre) et cela en fonction du sens de la baseline, défini par les sommets de départ et d'arrivée. Mais dans cette démarche toutes les baselines ont été numérisées à partir de la limite du côté terrestre de la zone tampon avec une distance de $150 \mathrm{~m}$ la séparant des shorelines. 


\subsection{Les calculs statistiques}

Cette étape s'est faite d'abord par la création de transects, puis par le calcul des indices et puis la représentation graphique des tables attributaires des indices calculés selon leur pertinence.

\section{Les transects}

Les transects sont des profils perpendiculaires à la ligne de base (baseline) qui permettent de mesurer la variation des taux d'évolution des traits côtes. Dans cette démarche, le nombre de transects est égal au nombre de profils utilisés et dépend de la longueur du segment (site) étudié. L'écart entre les profils mesurés automatiquement par DSAS est de $15 \mathrm{~m}$ sauf pour le site de Carabane $(10 \mathrm{~m})$ où le segment fait environ la moitié des autres segments. Cependant, il arrive très souvent sur la frange du littoral de la Casamance que certains transects apparaissent de manière aberrante suite à l'existence d'une ouverture d'embouchure ${ }^{1}$ au niveau du segment étudié. Or, les transects doivent couper tous les traits de côte qui lui sont perpendiculaires. D'autres sources de transects aberrants peuvent émaner de la sinuosité du linéaire côtier pouvant entraîner des saccades avant même leur intersection sur les shorelines. Il convient nécessairement de les corriger ou de les supprimer pour éviter les mesures inexistantes ou nulles.

\section{$\checkmark$ Calcul des indices}

Le calcul statistique consiste, en fonction des indices considérés, à faire ressortir les données tabulaires calculées automatiquement par DSAS. En effet, c'est l'analyse automatique de tous les transects qui permet de définir des secteurs d'évolution et d'en calculer les valeurs moyennes (Faye et al., 2010 ; Himmelstoss, 2009, Byrnes, 1994). Ainsi, après calcul, on obtient dans la table attributaire des transects les différents indices pris en compte lors de la configuration et dont les plus pertinents dans cette étude sont les End point Rate (EPR) et les Lineair Regression Rate-of-change (LRR).

\section{Résultats Cartographiques et Analyse Statistiques de l'évolution du Trait de Côte}

\subsection{Evolution du trait de côte au niveau du secteur de Diogué}

Le secteur allant de la «rivière aux huîtres » à la pointe de Diogué se situe sur la rive droite du fleuve Casamance, juste à l'embouchure. Le segment mesuré s'étend sur environ $10 \mathrm{~km}$.

\section{$\checkmark$ Évolution du trait de côte entre 1968 et 1986}

\footnotetext{
${ }^{1}$ Au niveau des embouchures il n'est pas possible de tracer un trait de côte entre les deux rives du fait de la rupture de la plage. Mais la baseline tient en compte et crée à ce niveau des transects de fausses mesures
} 
Durant la période 1968-1986, le secteur de Diogué a connu une accrétion au niveau de ses deux extrémités nord-ouest et sud-ouest, avec une marge d'incertitude de 1,66 m (fig. 2). Par ailleurs, au sud-est du village de Diogué, l'érosion s'est maintenue. Le nord du secteur qui correspond à la partie sud de l'embouchure de la « rivière aux huîtres » est caractérisé par une progradation atteignant une progression moyenne de $+150,30 \mathrm{~m}$, soit $+8,35$ $\mathrm{m} / \mathrm{an}$ en 18 ans. Le sud-ouest qui laisse voir les deux situations (érosion et accrétion) a dès lors connu globalement une accrétion moyenne de $+55,44 \mathrm{~m}$, soit $+3,08 \mathrm{~m} /$ an sur la même période. En revanche, au sud-est, l'érosion se manifeste avec un recul de $-145,08 \mathrm{~m}$ estimée à $-8,06 \mathrm{~m} / \mathrm{an}$. On peut noter que la période 1968-1986 est globalement caractérisée sur ce segment par une progradation, mais à certains endroits l'érosion a été active.

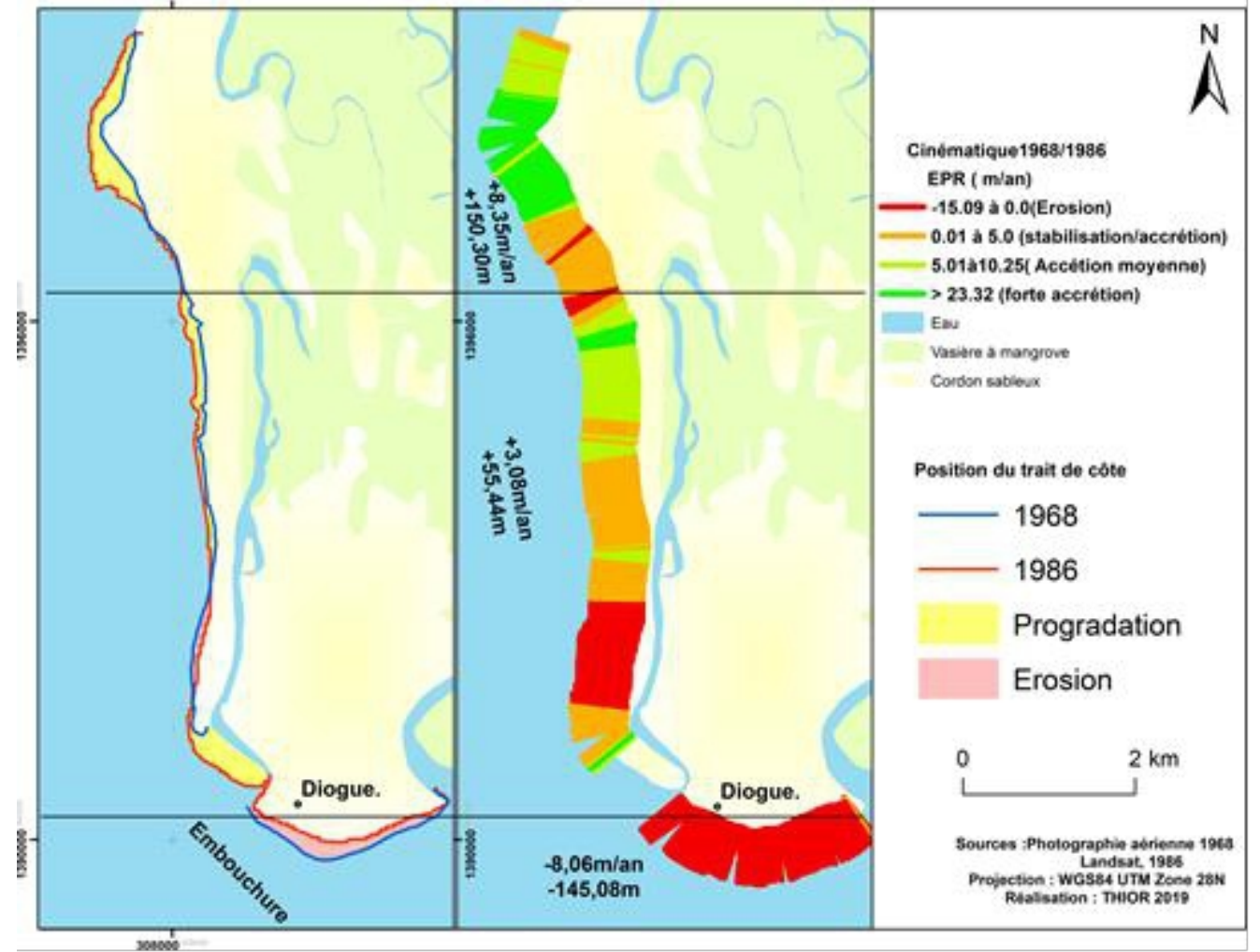

Figure 2: Évolution du trait de côte du secteur de Diogué entre 1968 et 1986

\section{$\checkmark$ Évolution du trait de côte entre 1986 et 2004}

La même tendance observée pendant la période précédente est similaire à celle de la période de 1989-2004 et avec les mêmes marges d'incertitude. Il s'agit globalement d'une progradation sur le segment avec quelques endroits de recul (fig. 3). Toutefois, les deux situations (érosion et progradation) se sont accélérées de part et d'autre. 


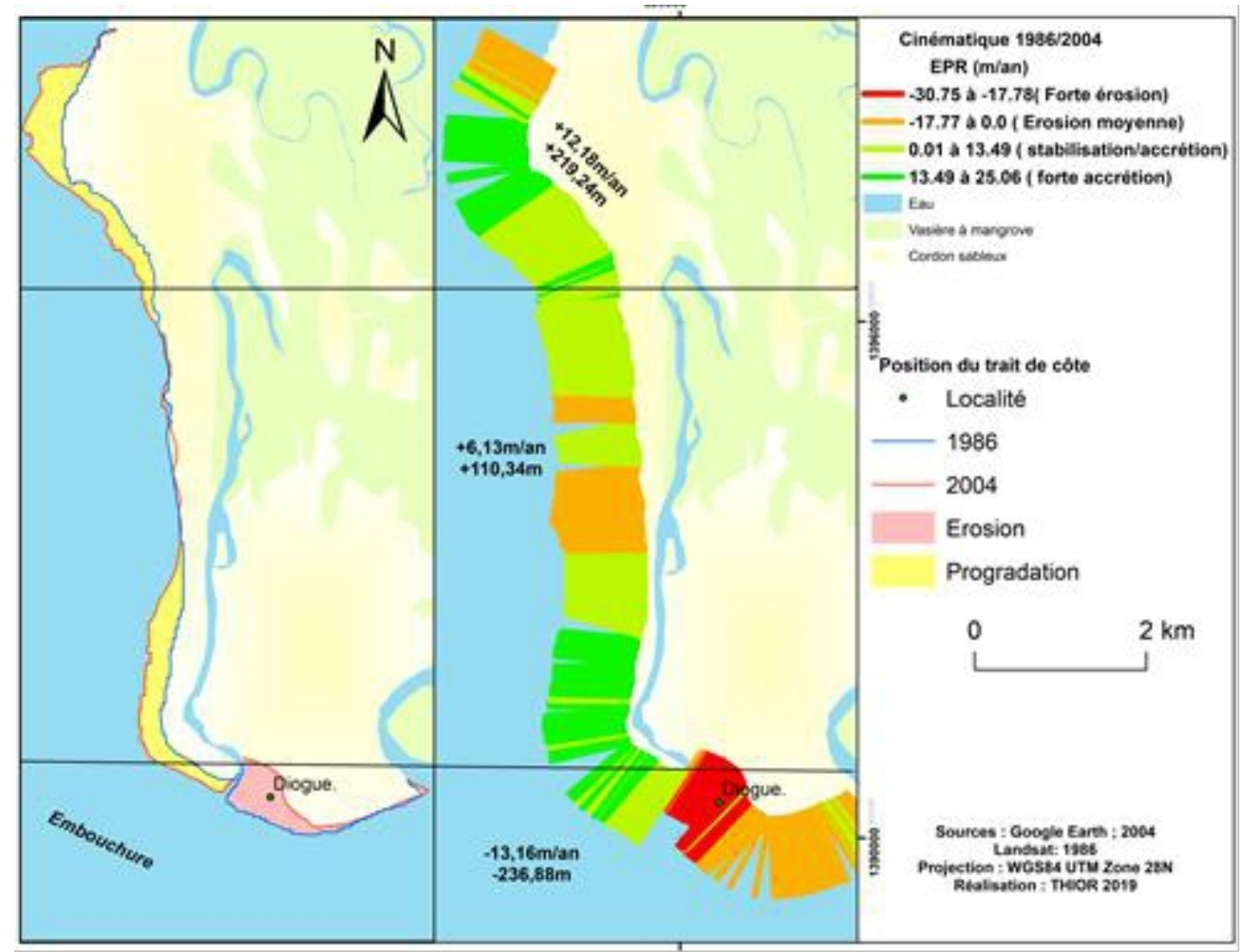

Figure 3: Évolution du trait de côte du secteur de Diogué entre 1986 et 2004

C'est en effet à l'image de la partie nord du secteur où la vitesse de progression passe de $+8,35 \mathrm{~m} /$ an entre 1968 et 1986 à $+12,18 \mathrm{~m} /$ an entre 1986 et 2004. Au sud-ouest, la vitesse de progression a doublé pour atteindre une moyenne de $+6,13 \mathrm{~m} / \mathrm{an}$. Inversement, le sud-est s'est érodé de $-13,16 \mathrm{~m} / \mathrm{an}$, soit un recul global de $-236,88 \mathrm{~m}$.

\section{Evolution du trait de côte entre 2004 et 2017}

La tendance évolutive est différente durant la période 2004-2017 avec une marge d'erreur moins importante $(0,7 \mathrm{~m} / \mathrm{an})$. En effet, la plupart des rives sud des embouchures de la zone ont connu un recul pendant cette période. Or, la partie nord du secteur constitue la rive gauche de l'embouchure de la «rivière aux huîtres ». Ce qui fait que les dépôts de la partie nord ont disparu en 13 ans, suite à la forte érosion de l'ordre de - 19,94 m/an contre une accrétion de $+12,12 \mathrm{~m} / \mathrm{an}$ sur la période précédente. En revanche, au sud du segment mesuré, la tendance reste la même avec une réduction de la vitesse d'évolution qui est de $+5,23 \mathrm{~m} / \mathrm{an}$, soit une progression moyenne de $67,99 \mathrm{~m}$. Par ailleurs, sur la partie sud-est, l'érosion continue avec un recul moyen de 49,27 m estimé à -3,79 m/an en 13 ans (fig. 4). 


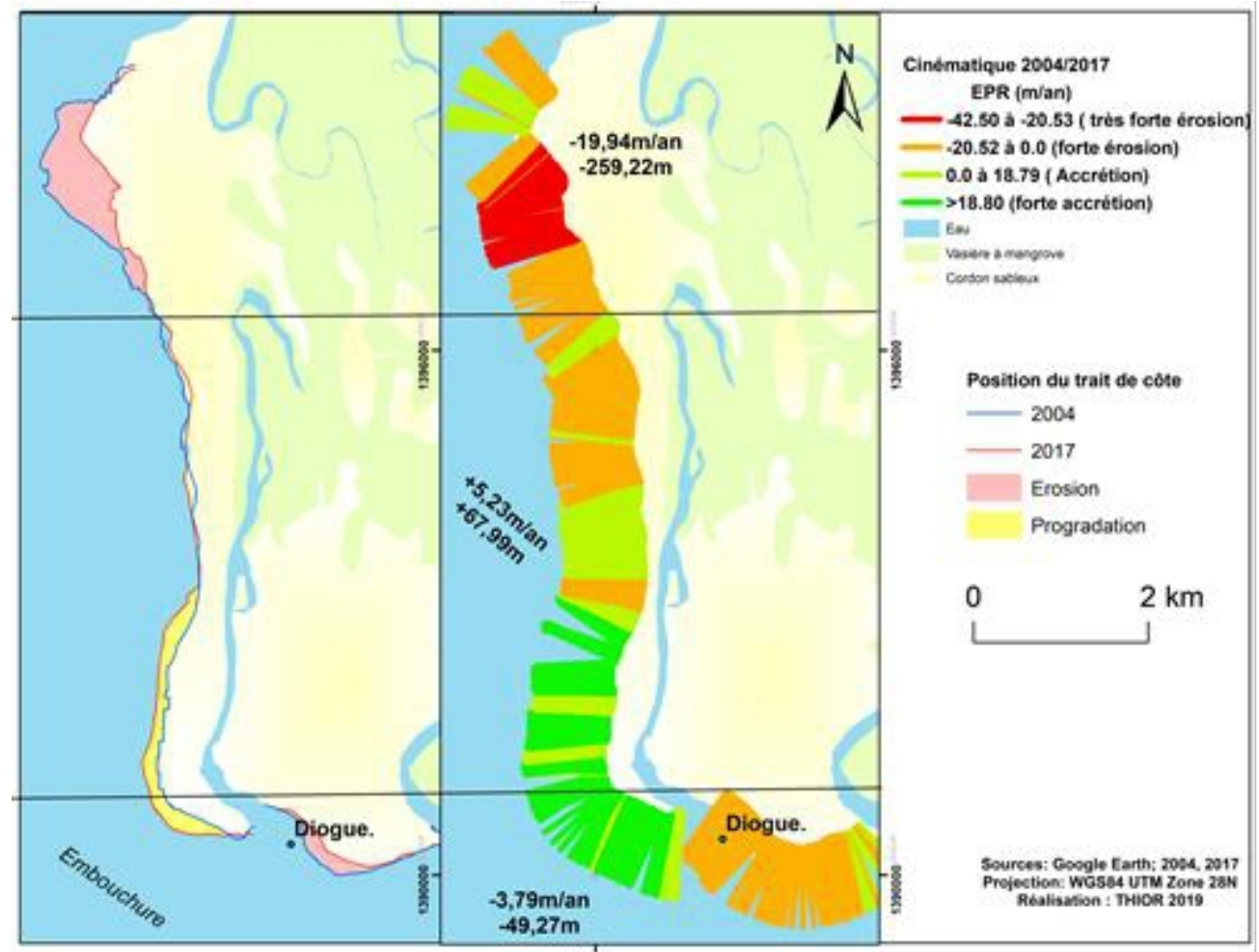

Figure 4: Évolution du trait de côte du secteur de Diogué entre 2004 et 2017

Les résultats de l'analyse des données de synthèse montrent une variabilité du LRR, indice qui permet d'apprécier la tendance qui se dégage sur toute la période étudiée. Il ressort dans ces résultats que le segment, entre la « rivière aux huîtres » et Diogué, n’est pas en équilibre, malgré les variations de la position du trait de côte (fig.5).

Par ailleurs, il faut noter que ce sont les pointes de flèches qui ont progressé et ont dissimulé les tendances de la position de la limite de la végétation. Néanmoins, au sud-est, où le recul est constant, il a entraîné une disparition d'une partie des rizières et une grande partie habitée par les pêcheurs. C'est la raison pour laquelle le bilan du recul moyen de cette partie est de $-11,22 \mathrm{~m} / \mathrm{an}$ sur toute la période étudiée. 


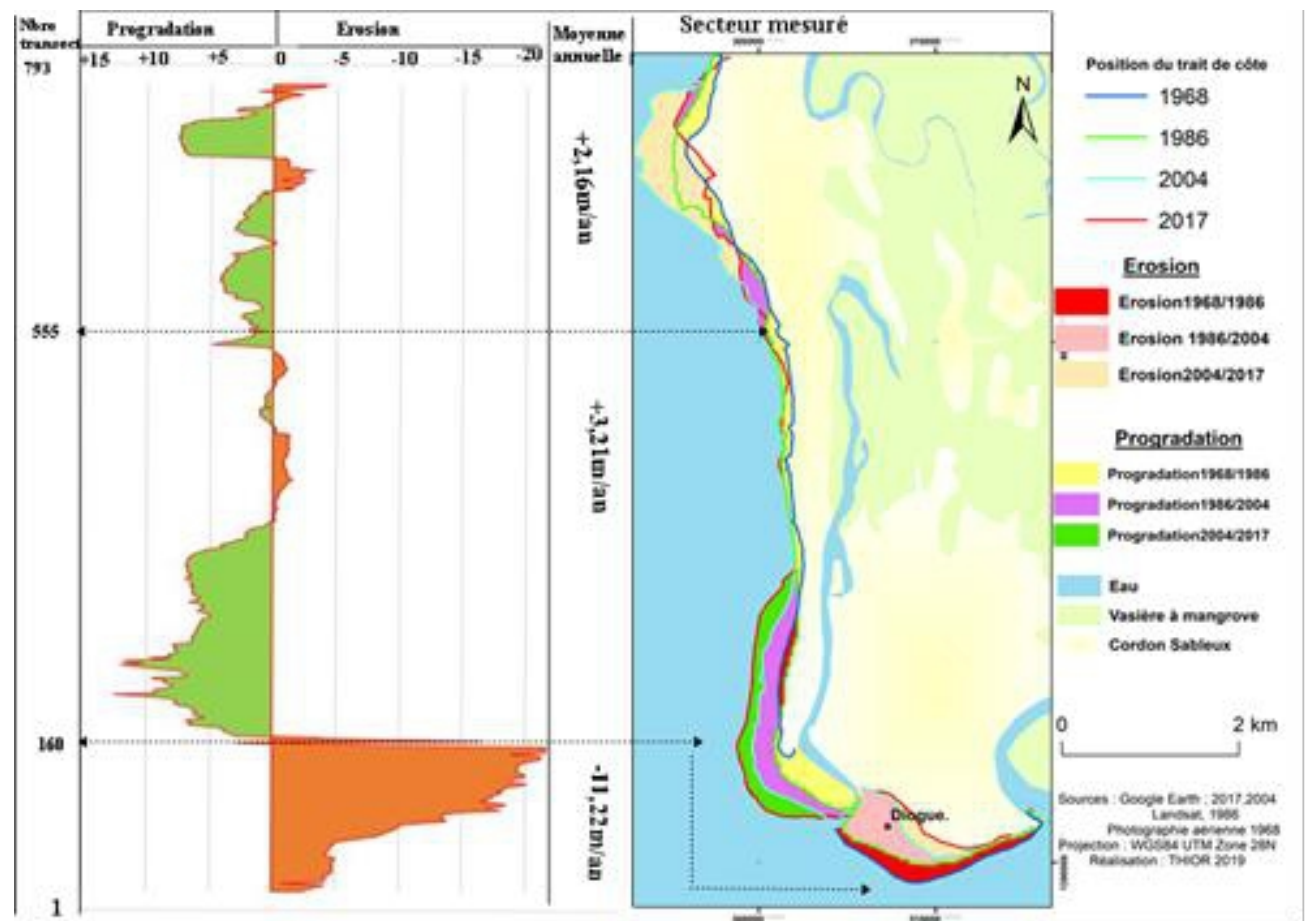

Figure 5: Synthèse et représentation graphique de l'évolution du trait de côte du secteur de Diogué entre 1968, 1986, 2004 et 2017

Au sud du secteur, le bilan de progression reste relativement important $\mathrm{du}$ fait de la sédimentation de la pointe de Diogué. C'est pourquoi on enregistre un taux de progression globale de $+3,21 \mathrm{~m} / \mathrm{an}$. Au nord du secteur, l'érosion entre 2004 et 2017 a dissimulé la forte progradation qu'a connue cette partie depuis 1968. De ce fait, la moyenne annuelle mesurée reste toujours progressive, mais relativement faible et elle est estimée à $+2,16 \mathrm{~m} / \mathrm{an}$.

On peut donc retenir sur le secteur de Diogué que, contrairement aux autres pointes qui progressent plus rapidement vers le sud, son rythme de progression ici est moins rapide. Cela s'explique par une progradation libre orientée vers l'ouest au fur et mesure qu'on s'approche de l'embouchure.

\section{2. Évolution du trait de côte du secteur de l'île de Carabane}

Ce secteur constitue la plus grande partie estuarienne du littoral de la Casamance et correspond particulièrement à la zone fluviomarine de l'île de Carabane. Siège des sédiments fins d'origine fluviale et marine, l'estuaire connaît une dynamique qui est propre et des mécanismes sédimentaires particuliers. L'apport des sédiments d'origine fluviomarine favorise le colmatage de l'estuaire, les accumulations de vasières latérales et de la construction de banc sableux médians (Mayer, 2006). Ainsi, la particularité du secteur étudié reste son ouverture sur l'embouchure où l'hydrodynamisme est 
similaire à celui de la frange côtière. Outre que cette particularité estuarienne, le segment mesuré est caractérisé par la présence d'une flèche sableuse de direction tantôt sud-nord tantôt ouest-est.

\section{$\checkmark$ Évolution du trait de côte entre 1968 et 1986}

La figure suivante indique l'évolution du trait de côte et la représentation statistique du secteur Carabane entre 1968 et 1986. Les mesures entre les deux traits de côte (entre 1968 et 1986) montrent une variation contrastée. C'est ainsi que, par endroits, on observe des phases d'érosion et des phases d'accrétion. En revanche, il faut noter que les variations de la limite supérieure de la végétation sont relativement faibles et dépassent légèrement les marges d'erreur. C'est au niveau de la pointe de la flèche qu'on observe une évolution importante de la variation du trait de côte.

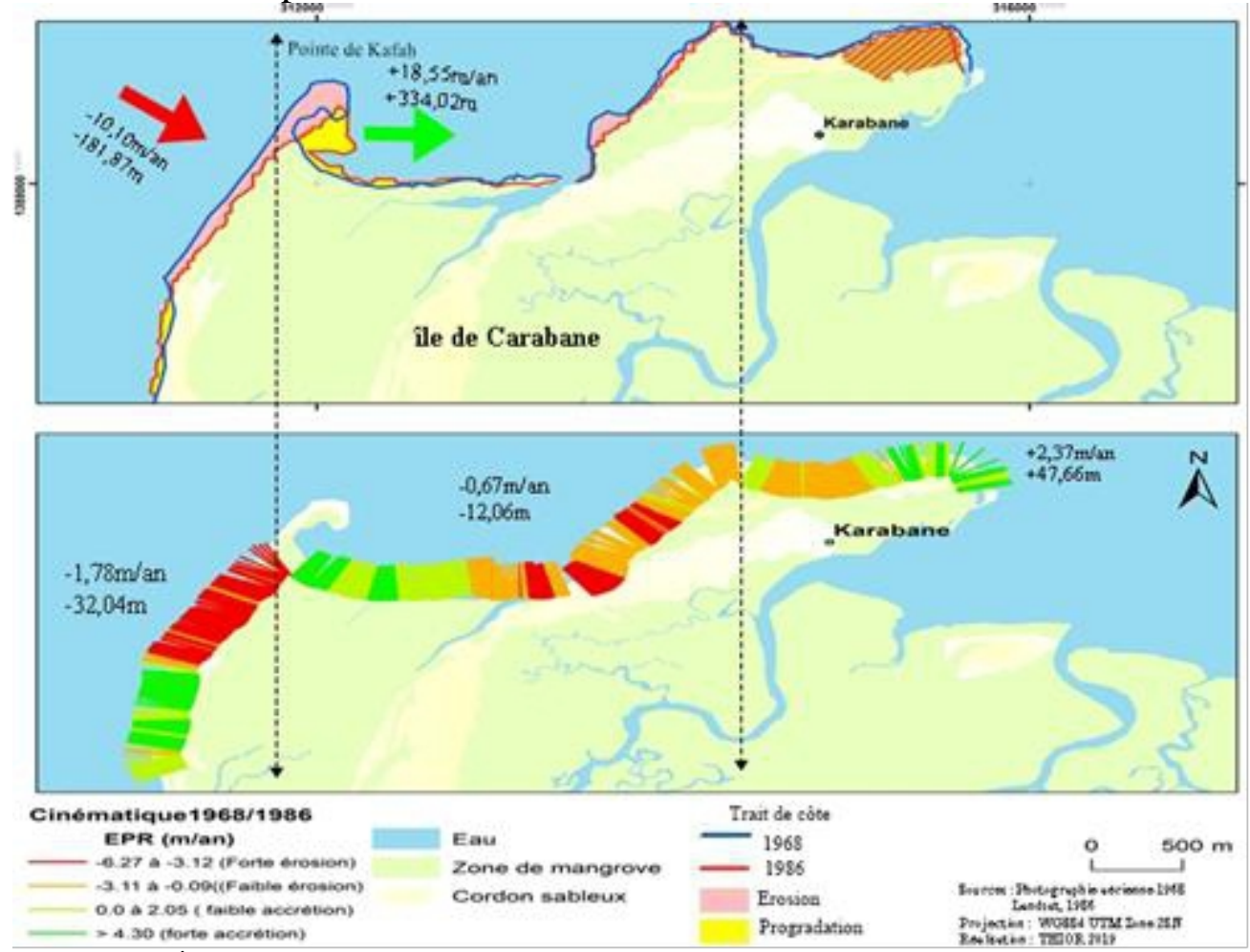

Figure 6: Évolution du trait de côte et représentation statistique du secteur Carabane entre 1968 et 1986

C'est pourquoi, dans la partie sud-ouest de l'île de Carabane, on enregistre, malgré la présence d'une accrétion, une érosion moyenne de $1,78 \mathrm{~m} / \mathrm{an}$, soit une perte linéaire de $-32,04 \mathrm{~m}$. Par ailleurs, à la pointe de la flèche de Kafah, l'érosion est accélérée avec un recul moyen de - 10,10 m/an estimé à 181,87 m sur une période de 18 ans. Dès lors, ce recul est compensé 
à l'est de la pointe et entraîne une modification significative de sa direction. La progression à l'Est est de $+334,02 \mathrm{~m}$, soit une progression de $+18,55 \mathrm{~m} / \mathrm{an}$ sur la même période.

Au nord du secteur, l'absence de flèche réduit la vitesse d'évolution du trait de côte. A cet effet, les écarts mesurés montrent globalement un recul de l'ordre de -0,67 m/an, soit une perte linéaire de 12,06 m entre 1968 et 1986. Ce qui est donc inclus dans l'incertitude, parce que ne dépassant pas la marge, tandis que la partie Est du secteur, moins exposé aux risques marins, connaît globalement une progression de $42,66 \mathrm{~m}$, soit $+2 \mathrm{~m} / \mathrm{an}$, même si par endroits on observe des phases d'érosion.

\section{$\checkmark$ Évolution du trait de côte entre 1986 et 2004}

A la différence de la première période, où on a constaté une érosion à la base de la pointe de Kafah et une progradation de celle-ci vers l'est, la période de 1986-2004 est caractérisée par une progradation de la flèche. Cette dernière s'est faite sur deux directions différentes. La première, orientée vers l'ouest, permet un développement de la largeur du cordon de la flèche, tandis que la seconde, en direction vers l'est, entraîne sa progression longitudinale (fig. 7).

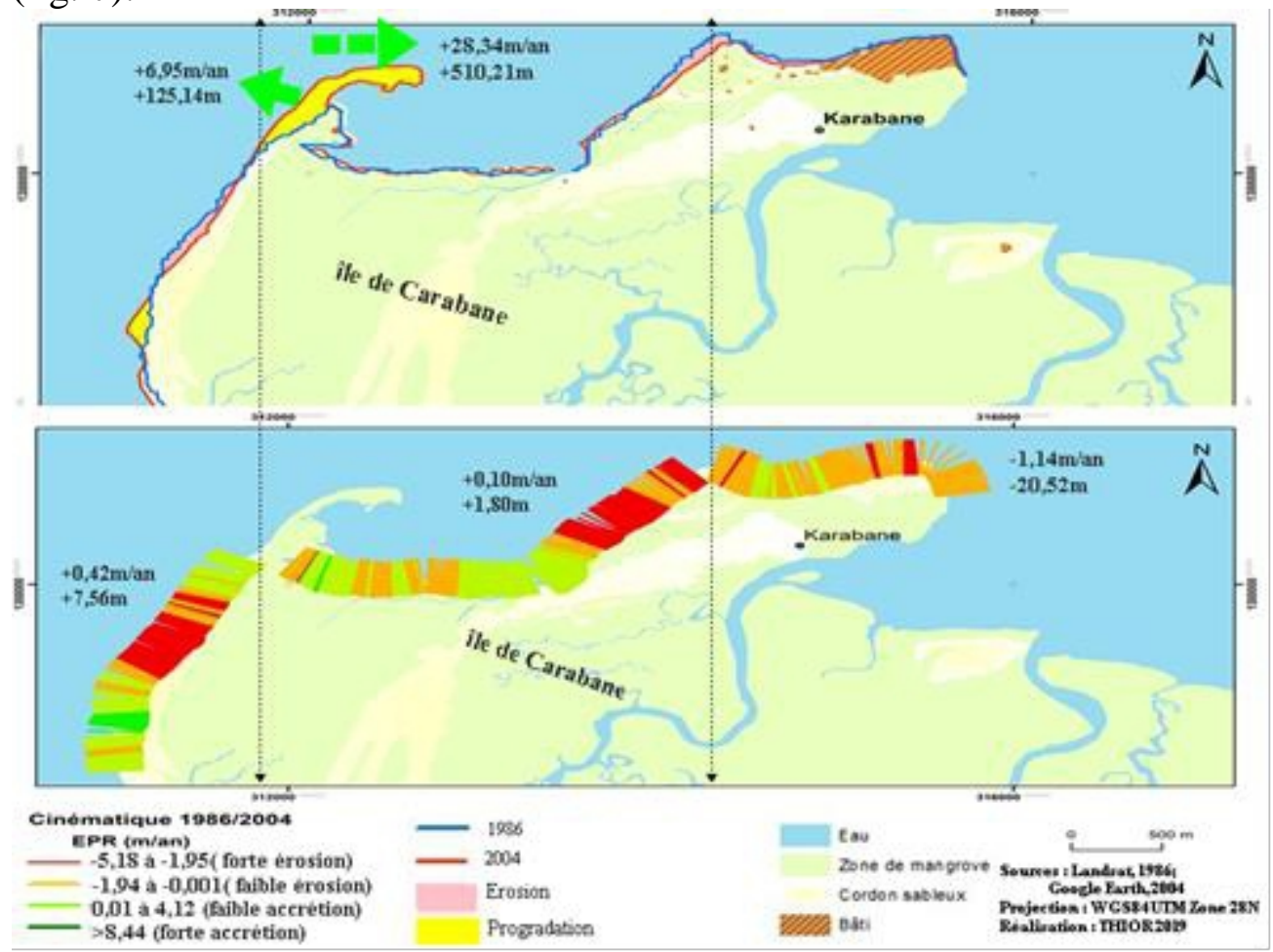

Figure 7: Évolution du trait de côte et représentation statistique du secteur Carabane entre 1986 et 2004 
Par ailleurs, au sud de cette accumulation de sable, se manifeste une érosion significative qui explique la sédimentation au nord. Par conséquent, les mesures de l'indice EPR entre les traits de côte montrent une augmentation de l'épaisseur de la pointe de Kafah estimée à $+6,95 \mathrm{~m} / \mathrm{an}$ en moyenne, soit 125,14 m entre 1986 et 2004, alors que la progression vers l'Est laisse voir une vitesse moyenne plus rapide. En effet, cette vitesse est de $+28,34 \mathrm{~m} / \mathrm{an}$ en moyenne. Elle permet alors à la pointe de progresser de 510,21 m vers l'Est sur la période observée, soit en direction de l'amont du fleuve Casamance.

Outre cette évolution individuelle du segment étudié, les mesures sur les transects montrent que dans la partie sud-ouest, l'évolution de la position de la limite de la végétation a relativement progressé, mais avec des endroits érodés. Cette progression n'est pas significative puisque le travail de l'érosion ralentit cette progression, et le résultat statistique est compris entre la marge d'incertitude.

Au nord du segment, le secteur est relativement en équilibre, car il ne progresse que de $+1,80 \mathrm{~m}$, soit une accrétion de $+0,10 \mathrm{~m} / \mathrm{an}$, ce qui ne peut être considéré puisqu'inclus dans la marge d'erreur. De la même manière, les résultats dans la partie Est, où la vitesse de l'érosion est bien importante, parce qu'elle atteint en moyenne $-1,14 \mathrm{~m} / \mathrm{an}$, mais toujours interprétable, sont compris dans la marge d'incertitude.

Cette partie du secteur semble être la partie la moins exposée en raison de la protection naturelle de la pointe de Kafah, mais aussi de la présence du banc de sable à l'Est. Par conséquent, la vitesse des courants de transport sédimentaire est réduite par ces deux extrémités.

\section{$\checkmark$ Évolution du trait de côte entre 2004 et 2017}

Concernant la période 2004-2017, on note des plages en équilibre le long du segment. Ainsi, la zone dynamique reste le cordon et la pointe de la flèche sableuse (fig. 8). 


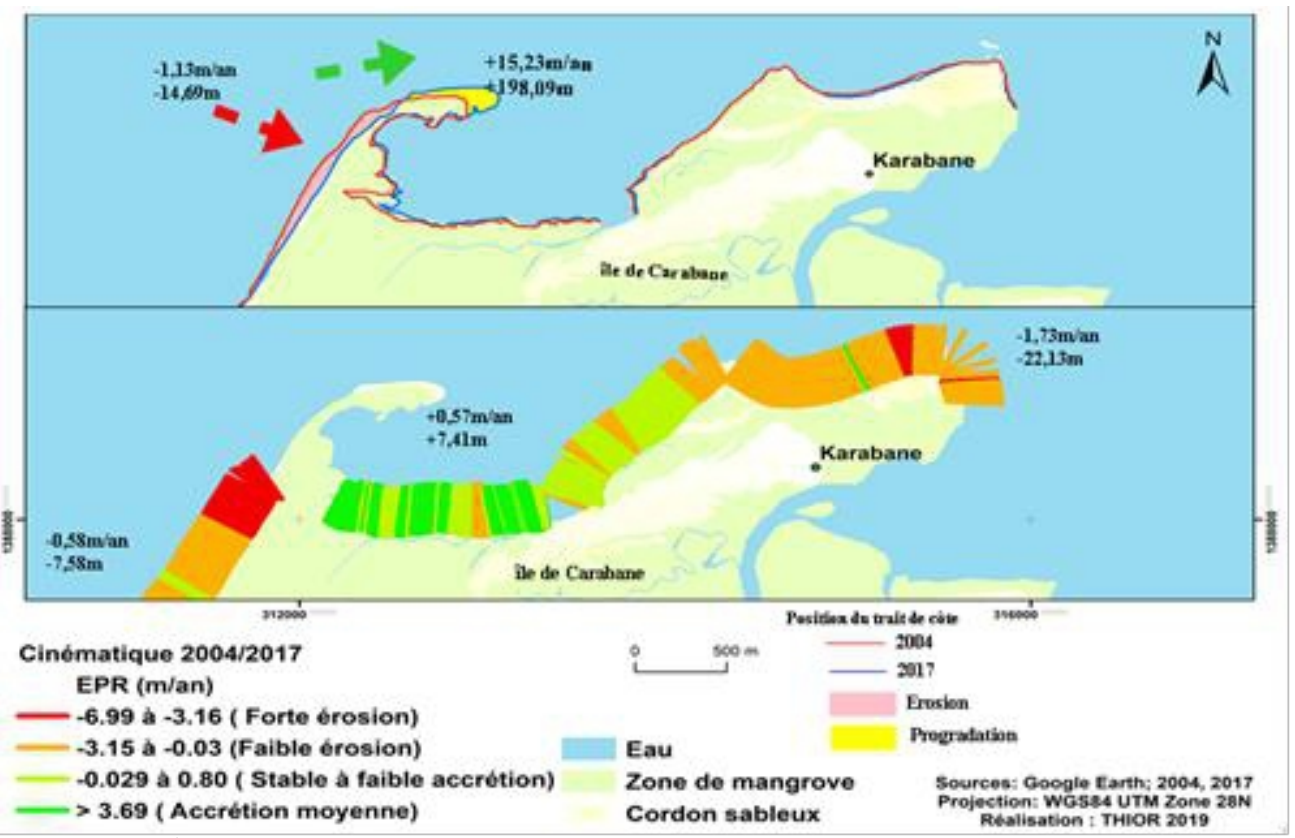

Figure 8: Évolution du trait de côte et représentation statistique du secteur Carabane entre 2004 et 2017

La flèche prend définitivement une direction ouest-est avec une forte érosion de sa partie sud-ouest. En plus, la vitesse d'érosion à ce niveau fait que l'ensemble du système paysager de la flèche roule sur elle-même et entraîne un recul considérable de plage. Avec un recul de - 1,13 m/an en moyenne, le cordon de la flèche a reculé de $-14,69 \mathrm{~m}$ sur 13 ans. Ce cordon qui mesurait $25 \mathrm{~m}$ de largeur en 2015 est de $15,75 \mathrm{~m}$ en janvier $2018 .^{2}$

À ce rythme d'érosion, une possible ouverture naturelle risque de se produire et créera une rupture de la flèche. Par ailleurs, le colmatage de ces sédiments arrachés à ce niveau puis déposés à la pointe lui donne une orientation ouest-est. Cette progression de la pointe sableuse est de $+198,09 \mathrm{~m}$, soit une moyenne de $+15,23 \mathrm{~m} / \mathrm{an}$. Cependant, malgré les modifications notées sur la pointe de Kafah entre 2004 et 2017, les statistiques sur l'EPR montrent que l'extrémité sud-ouest est en très faible érosion et n'a reculé en moyenne que de $-7,58 \mathrm{~m}$, soit un recul moyen de $-0,58 \mathrm{~m} / \mathrm{an}$. Ce même rythme de recul est inversé au nord du segment en aval de la pointe avec une légère différence et il est de $+7,41 \mathrm{~m}$, soit une progression moyenne de $+0,57 \mathrm{~m} / \mathrm{an}$.

En revanche, à l'est du segment mesuré, l'érosion s'est accélérée, mais avec une durée moins longue que celle qui l'a précédée. En effet, au cours de cette période, des infrastructures ont été installées modifiant

${ }^{2}$ Mesures effectuées lors d'une descente particulière sur le terrain pour voir le niveau de vulnérabilité de la flèche. 
l'hydrodynamisme naturel. Il s'agit du ponton et de ses extensions qui font que la plage à ce niveau ne recule pas de manière homogène. De ce fait, ces infrastructures de protection créent des mécanismes qui freinent le courant qui doit transporter le sable vers les plages voisines (ouest et est de l'infrastructure). Par conséquent, l'équilibre est à ce niveau rompu, car il part davantage plus de sable dans large qu'il n'en arrive au rivage. Ainsi, on enregistre un recul en moyenne de $-1,73 \mathrm{~m} / \mathrm{an}$, soit une régression moyenne de $-22,13$ m en 13ans.

En effet, au cours de toute la période étudiée, l'indice de LRR calculé a montré que le littoral fluviomarin de Carabane a connu des phases d'érosion et d'accrétion. Ainsi, la partie sud-ouest de l'île est marquée par un léger bilan positif, malgré la forte érosion du cordon de la flèche. Cette faible progression est de $+0,42 \mathrm{~m} / \mathrm{an}$, tandis qu'au nord du secteur la progression de la pointe de Kafah vers l'est a dissimulé les rares endroits érodés. Par conséquent, le bilan de l'évolution est de $+1,07 \mathrm{~m} / \mathrm{an}$. En revanche, dans la partie nord-est, le bilan reste globalement négatif avec un recul en moyenne de $-0,91 \mathrm{~m} / \mathrm{an}$ (fig. 9).

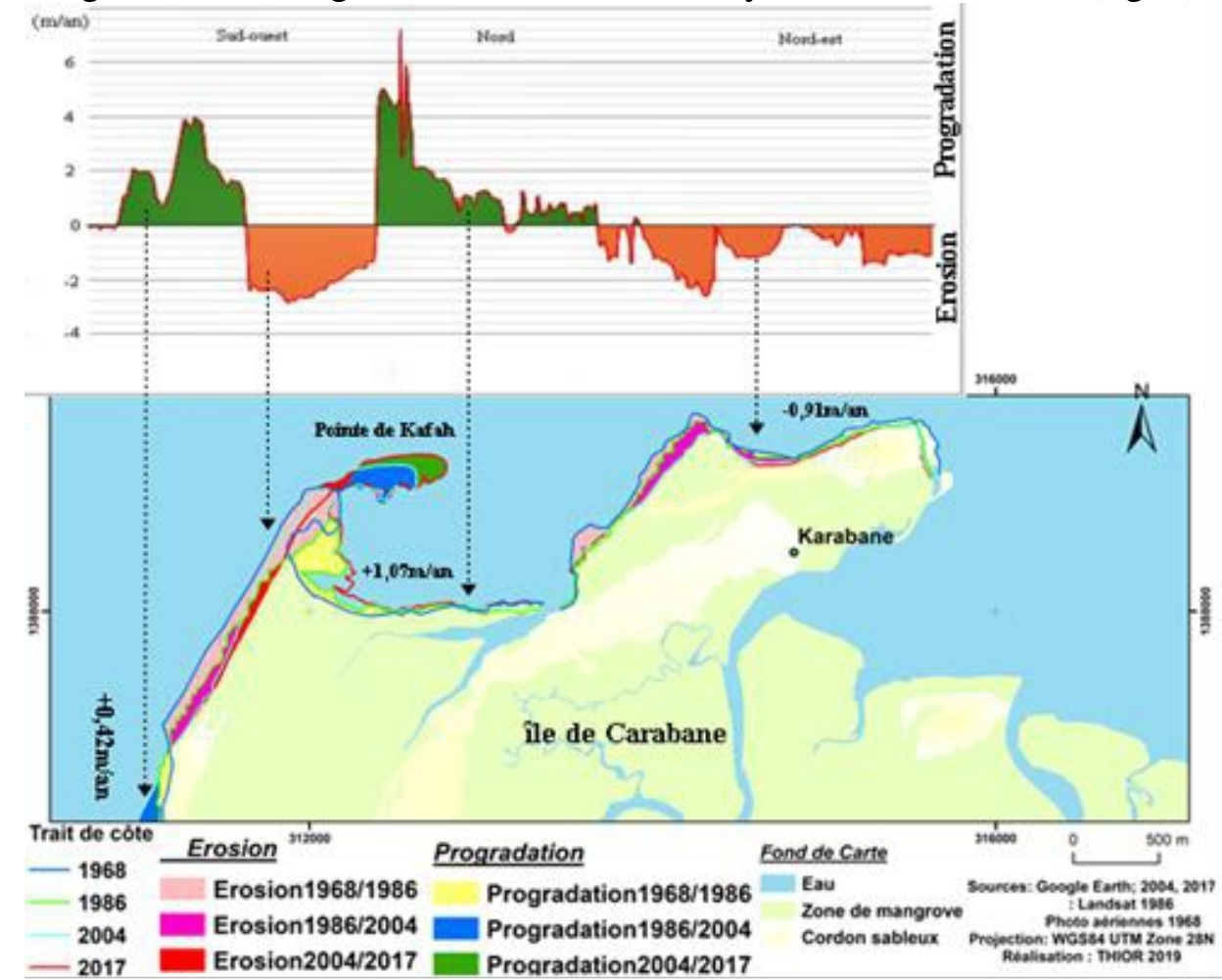

Figure 9: Représentation graphique de la synthèse de l'évolution moyenne de trait de côte sur toute la période

\section{3. Évolution du trait de côte du secteur de Gnikine-Diembéring}

Le transect Gnikine-Diembéring constitue la partie sud de l'embouchure du fleuve Casamance. À l'instar des autres secteurs du nord de 
l'embouchure, ce secteur reste sous l'influence de la houle longue du Nordouest. Cela se manifeste par un recul permanent de la pointe nord de Gnikine et l'absence globale de dépôts sableux pouvant entraîner un possible développement de flèche littoral.

\section{$\checkmark$ Évolution du trait de côte entre 1968 et 1986}

La période 1968 -1986 est caractérisée par une tendance globalement érosive (fig. 10). Néanmoins, avec une marge d'incertitude de $1,66 \mathrm{~m}$, on peut considérer que les résultats statistiques sont interprétables.

Au nord du segment mesuré, la limite de la position de la végétation a connu un recul en moyenne de $-54,90 \mathrm{~m}$, soit une perte de $-3,02 \mathrm{~m} / \mathrm{an}$. Au centre-ouest, le recul s'accélère avec un recul moyen de $-7,59 \mathrm{~m} / \mathrm{an}$, tandis qu'au sud la côte est pratiquement en équilibre. En effet, même si au sud du secteur mesuré, on enregistre par endroits de légers taux d'érosion, l'accrétion y domine relativement. Ce qui donne une plage qui progresse néanmoins de $+0,06 \mathrm{~m} / \mathrm{an}$, soit une accrétion moyenne de $+1,08 \mathrm{~m}$ en 18 ans. Or, l'incertitude est de 1,66 m. Ce qui laisse cependant observer une plage en équilibre.

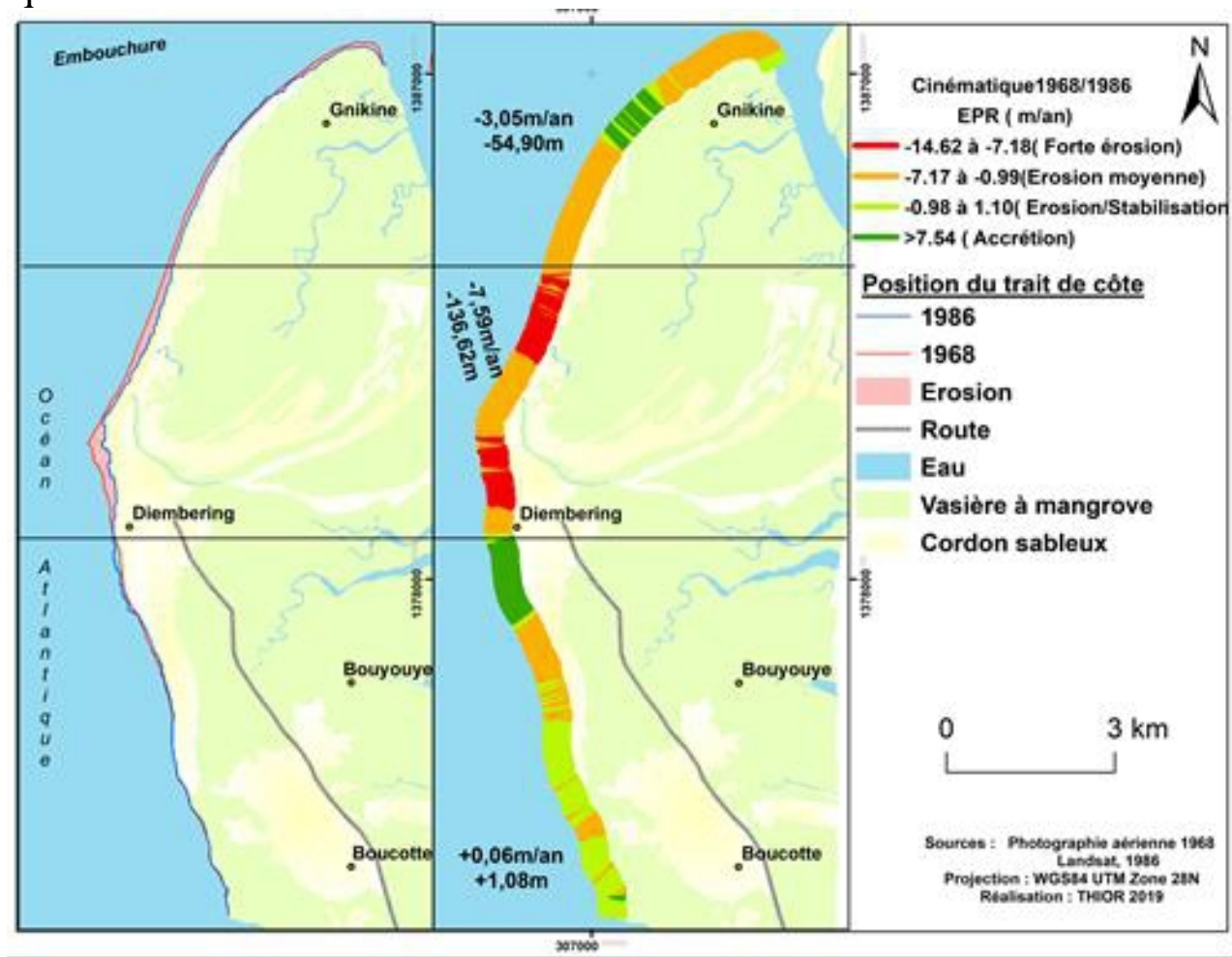

Figure 10: Evolution du trait de côte et représentation statistique du secteur de Gnikine Diembéring entre 1968 et 1986 


\section{$\checkmark$ Évolution du trait de côte 1986 et 2004}

La période entre 1986-2004, qui correspond généralement à la période où l'essentiel du littoral de la Casamance a connu un recul, se manifeste ici par une plus forte érosion au nord de la pointe de Gnikine (fig. 11).

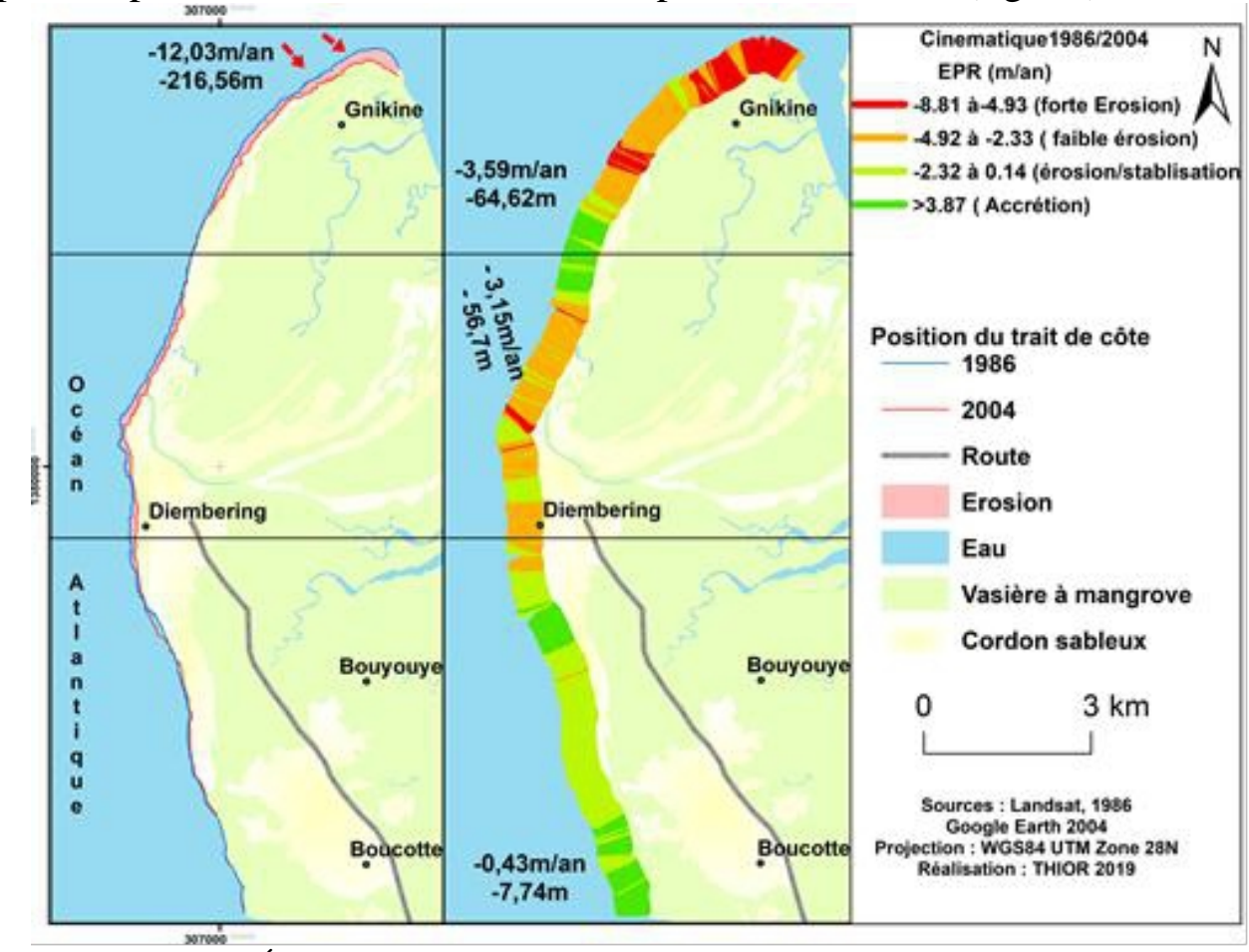

Figure 11: Évolution du trait de côte et représentation statistique du secteur de Gnikine - Diembéring entre 1986 et 2004

Evidemment, sur le plan morpho sédimentaire, les deux tendances ${ }^{3}$ qui se dégagent sur les côtes ouest-africaines (influence de la houle du Nord-ouest et de celle du Sud-ouest) sont en faveur d'une domination du courant de la dérive nord-sud (Diop., 1990). C'est pourquoi il est rare que la pointe sud de l'embouchure du fleuve Casamance progresse vers le nord de la pointe. C'est pourquoi la pointe a reculé de $+216,56 \mathrm{~m}$ entre 1986 et 2004, soit une progression moyenne de $+12,03 \mathrm{~m} / \mathrm{an}$. Cependant, la moyenne sur la partie nord est estimée à $-3,59 \mathrm{~m} / \mathrm{an}$. Cette vitesse d'érosion est plus ou moins similaire à celle observée au centre ouest avec un recul en moyenne de $3,15 \mathrm{~m} / \mathrm{an}$, soit une perte de $-56,70 \mathrm{~m}$, tandis qu'au sud du segment, le très

3 - D'une part, une remarquable influence de la houle du sud-ouest jusqu'à des latitudes très septentrionales, notamment en saison des pluies, mais aussi un effet prépondérant de cette même houle dans l'envasement des «bouches» de certains estuaires (Salonm, Kapatchez, Somba etc.) et dans l'alimentation des vasières situées au large, sur le plateau continental. (Gambie, Casamance...). D'autre part, une prédominance de la houle du nord-ouest en saison sèche jusque dans le nord de la Guinée Bissau et même au large de Conakry (Diop1990). 
faible taux d'érosion (-0,43 m/an, marge d'incertitude) laisse toujours cette partie en équilibre.

\section{$\checkmark \quad$ Évolution du trait de côte entre 2004 et 2017}

La période 2004-2017 est caractérisée par un recul globalement faible comparé au littoral nord de la Casamance. C'est pourquoi, du nord au sud, la vitesse moyenne du taux d'érosion diminue. A ce propos, le calcul de l'indice de L'EPR montre néanmoins une érosion plus accentuée au nord comme d'ailleurs pour les périodes précédentes.

Malgré l'échelle temporelle (13 ans), le taux de recul est similaire au nord à celui de la période précédente (18 ans), soit -3,59 m/an en 1986 et 2004 contre -3,45 m/an entre 2004 et 2017 (fig. 12). Par ailleurs, au centre ouest du secteur mesuré, le taux de recul moyen est réduit suite à une stabilisation d'une partie de la plage. C'est la raison pour laquelle on enregistre une perte linéaire de $-32,63 \mathrm{~m}$ en moyenne, soit un taux de recul de 2,51 m/an. Cependant, le sud du segment mesuré a connu une accélération de sa vitesse d'érosion avec une perte de $-14,69 \mathrm{~m}$ en moyenne, soit une érosion de 1,13 m/an en 13 ans correspondant au double de la période précédente et plus longue. A cet effet, en considérant la marge d'incertitude très faible comparée aux périodes précédentes, on peut interpréter les résultats statistiques.

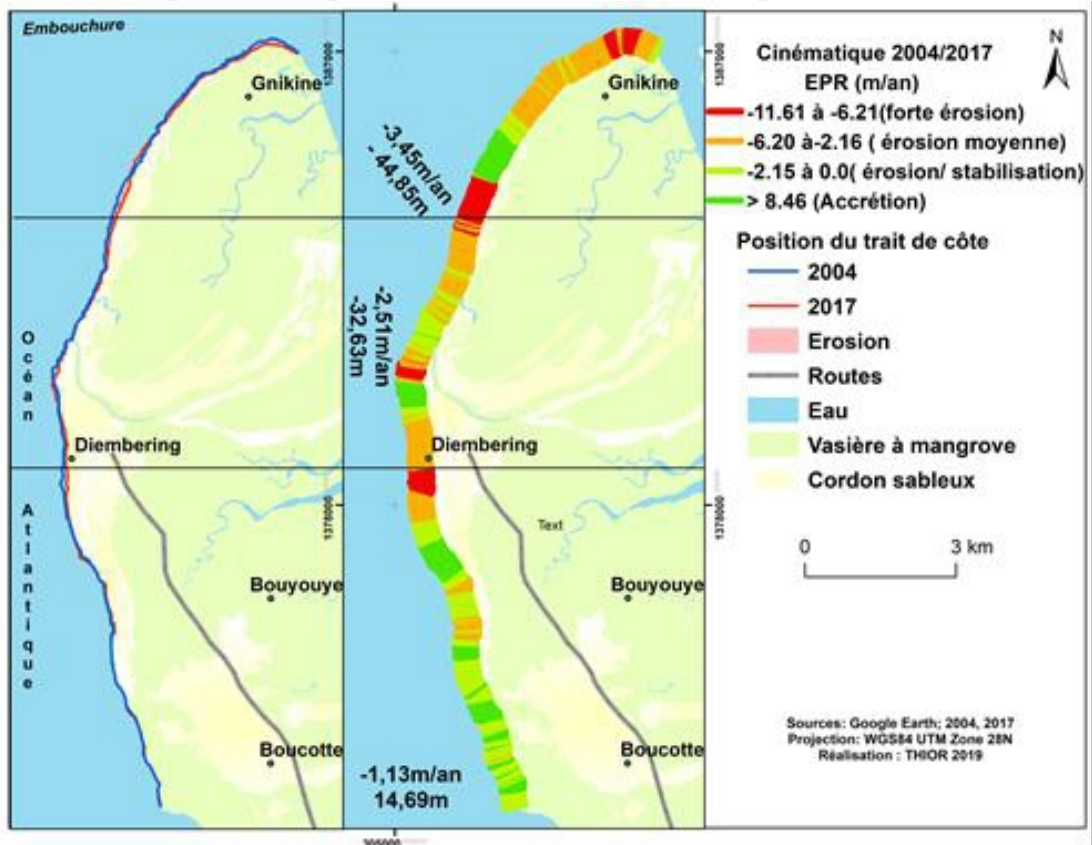

Figure 12: Evolution du trait de côte et représentation statistique du secteur de GnikineDiembéring entre 2004/2017

Au total, l'analyse des trois périodes sur l'évolution du trait de côte obtenue par le calcul du LRR montre que la tendance globale sur le secteur de 
Gnikine-Diembéring est érosive. En effet, l'érosion s'accentue au fur et à mesure que l'on avance vers l'embouchure du fleuve Casamance. Cela s'explique en grande partie par le fait qu'au sud du segment, la présence du plateau continental laisse apparaître un typique modelé du grès ferrugineux ou Beach rocher, permettant à la plage qui les porte de rester à l'abri d'une forte érosion. Ces roches jouent un rôle de protection similaire à celui d'une accumulation de galet. Selon Morel (1999), les accumulations de galets sont des formations meubles qui ajustent leur morphologie aux conditions hydrodynamiques du moment. De ce fait, elles ont une capacité à s'adapter aux différentes contraintes hydrodynamiques, et tout particulièrement aux tempêtes qui leur vaut la réputation de « tampon » protecteur naturel des côtes. Les accumulations qui se développent devant des zones basses aux altitudes inférieures au niveau des pleines mers jouent le rôle de barrière antisubmersion, tandis que celles qui se forment au pied des falaises remplissent le rôle de pare-chocs contre les coups de boutoir des vagues (Mayer, 2006). Par ailleurs, au nord, en plus de l'influence de la houle du Nord-ouest plus longue au cours de l'année, la côte est caractérisée par des systèmes dunaires, dont la structure est meuble. La combinaison de ces deux éléments laisse la côte vulnérable face à l'érosion marine. Ce qui fait qu'on observe une érosion moyenne annuelle de $-4,32 \mathrm{~m} / \mathrm{an}$ sur l'ensemble de la période (fig. 13).

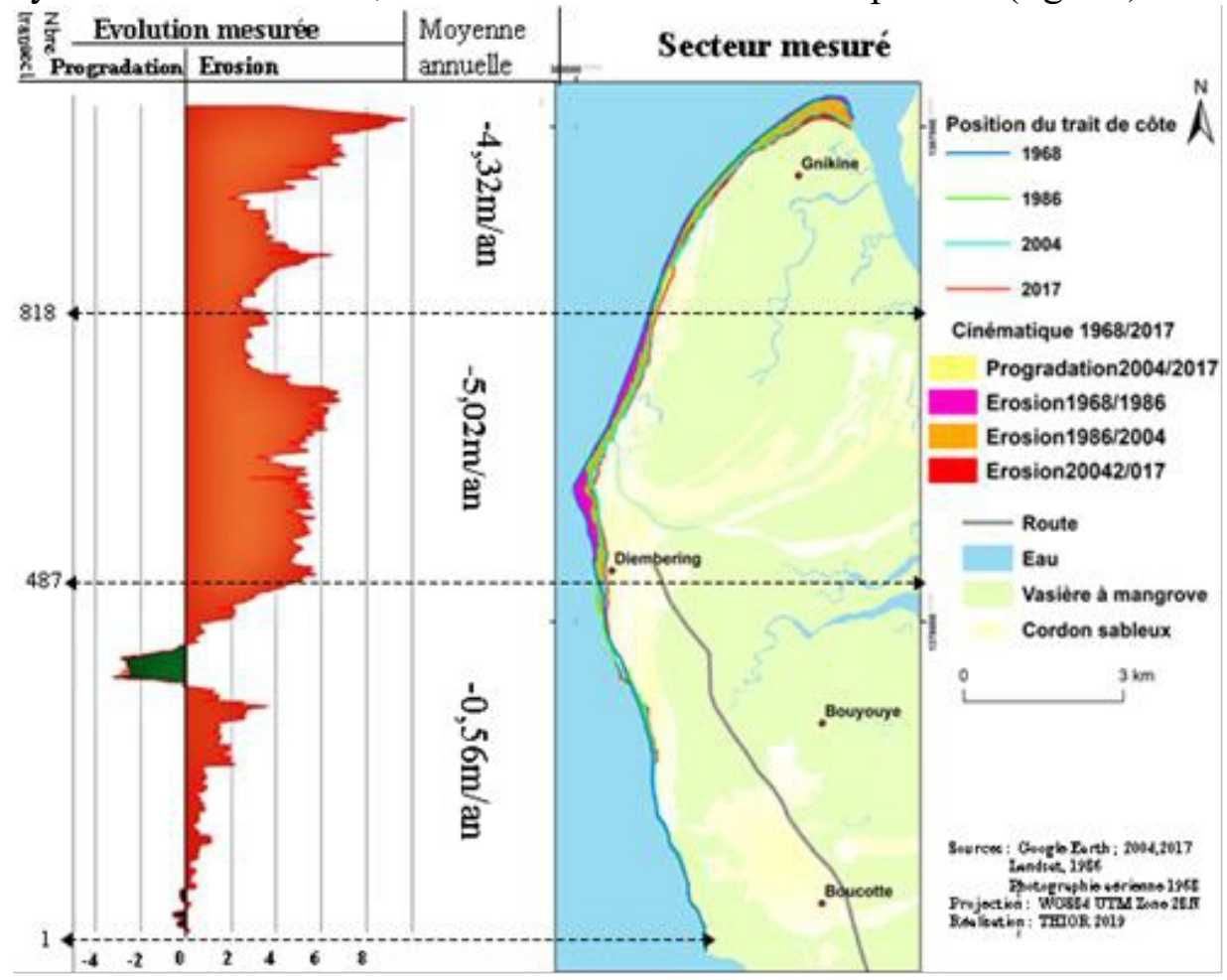

Figure 13: Synthèse de l'évolution moyenne du trait de côte du secteur Gnikine-Diembéring entre 1968 et 2017 
Au centre-ouest du secteur, le bilan de l'évolution du trait de côte est également caractérisé par un recul, dont le taux en moyenne est de -5,02 m/an. Cela est perceptible par l'érosion d'un front de mer pendant la période 1968/1986 (fig.13).

Le sud du secteur mesuré, compris entre le transect 1 et le transect 487, montre que la tendance évolutive du trait de côte est légèrement érosive, mais la faiblesse de la vitesse de l'érosion laisse voir une plage en équilibre. Ainsi, malgré la présence faible de zones de progradation dans cette partie, on observe un bilan érosif avec un taux de -0,56 m/an (fig.13).

On peut globalement noter que le secteur de Gnikine-Diembéring, contrairement aux côtes du nord qui ont connu des phases d'érosions et des phases de progradation, a connu au cours d'un demi-siècle (1968/2017) une érosion globale de sa plage.

\section{Discussions}

Naturellement, trois situations sont observées sur le littoral de la Casamance : il s'agit de stabilisation, érosion et accrétion. L'objectif de cette étude était de montrer la mobilité du trait de côte à travers ces trois situations. En plus de ces situations, les orientations des progressions ou régressions sont conditionnées par des agents hydrodynamiques (dérive littorale, courant de la marée). Les plages ont toujours fonctionné suivant la compétence des vents, de la dérive littorale nord-sud (Niang, 2017; Rue, 2005). L'analyse des résultats cartographiques issus de la cinématique considérée par Pennober (1999) comme l'étude des formes indépendantes des forces qui en sont les causes. Mais il existe plusieurs démarches pour suivre la dynamique du trait de côte. Ainsi, la diversité des démarches méthodologiques fait que plusieurs auteurs peuvent travailler sur une même thématique avec des approches différentes et arriver à des résultats similaires. L'analyse spatiale peut donc être appliquée sur presque tous les domaines. Mais l'idéal en cinématique du littoral est de disposer d'une série d'images ayant des conditions météo marines identiques. Or, il est très rare de tomber sur un document historique où ces informations sont mentionnées. C'est pourquoi il est plus prudent de travailler avec des indicateurs de trait de côte indépendants du type de marée, lorsque les côtes sur lesquelles porte l'analyse spatiale présentent des lignes de référence physiques. ${ }^{4}$

L'application de cette démarche sur le littoral de la Basse Casamance suppose d'abord que l'on dispose de photographies aériennes anciennes et d'images satellitaires multi dates et de documents cartographiques anciens ; les premières devant être les plus vieilles parmi celles dont l'exploitation est aisée. Mais les résultats de cette superposition sont souvent discutés du fait

\footnotetext{
${ }^{4}$ Les limites géomorphologiques ou les limites de la végétation
} 
des erreurs liées à la résolution des images (Landsat), à la saisie du trait de côte, aux erreurs de géoréférencement (RMS). C'est pourquoi, on peut remarquer une différence entre les résultats des travaux de Faye (2010), même si les tendances sont globalement similaires, mais avec des pas de temps inégaux. De ce fait, en comparant les résultats des taux de recul à la pointe de Gnikine, on trouve un recul de $-12,03 \mathrm{~m} / \mathrm{an}$ entre 1986 et 2004 et $-12,22 \mathrm{~m} / \mathrm{an}$ pour Faye (2010) entre 1986 et 2000. Cependant, pour notre cas, ce taux de recul ne concerne que la pointe qui ne prend pas en compte le référentiel du sud de la pointe (limite de la végétation). Le recul moyen du secteur est donc de $-3,59 \mathrm{~m} / \mathrm{an}$. Il en est de même pour la pointe de Kafah (Carabane) où la dynamique est plus importante sur la pointe que sur les rivages. Sous ce rapport, les tendances sont également similaires et les résultats sur la pointe sont approximatifs. En revanche, l'évolution sur la plage n'est pas précisée par Faye (2010) mais est de -0,42 m/an au nord-ouest de 1986 à 2004. Cette valeur reste alors bien à discuter puisqu'elle est comprise entre la marge d'erreur qui est de 1,66 m du fait de la résolution de l'image Landsat de 1986. Par ailleurs, comparées aux résultats de Barry (2016), les tendances dans les deux cas restent plus ou moins similaires même si les échelles temporelles sont plus longues dans notre étude. Cependant, les mesures de Barry basées sur une approche purement télédétection n'ont pas précisé les marges d'erreur.

\section{Conclusion}

En cinématique du littoral, le traitement numérique de l'information géographique est presque devenu une démarche standard bien que des améliorations de la technologie d'analyse spatio-temporelle soient en perpétuelle avancée. Ainsi, le recours à des méthodes statistiques d'extrapolation ou de calcul de tendance est souvent utilisé en matière d'étude de la dynamique du trait de côte, à travers les outils d'analyse spatiale. Il apparaît donc que plusieurs données soient utilisées dans l'analyse de l'évolution du trait de côte. Pour le littoral de la Casamance, nous disposons de la photographie aérienne de 1968, de l'imagerie Landsat de 1986 et des images de Google Earth de 2004 et 2017. Alors, chaque image favorise la pertinence d'un type de ligne de référence. En plus, les données géospatiales n'ont pas la même résolution. Les résultats d'une telle superposition d'images peuvent susciter des marges d'erreur. Mais pour une embouchure où la dynamique est grandiose, la correction des erreurs peut être intégrée dans l'analyse. Or, les résultats et les connaissances du terrain révèlent une côte très dynamique à l'embouchure de la Casamance. En effet, siège des sédiments fins d'origine fluviatile et marine, l'embouchure de la Casamance connaît une dynamique qui lui est propre et des mécanismes sédimentaires particuliers. Sous l'influence du courant de la dérive induite par la houle du nord-ouest, la pointe de Diogué a connu entre 1968 et 1986 une érosion en amont et au sud- 
est. Tandis que, le Sud-ouest est caractérisé par une progradation vers le, sud. La rive sud de l'embouchure connaît par contre un recul moins fort sur le front nord et une légère stabilisation par endroit. A l'est, l'île de Carabane est caractérisée par la présence de pointe de Kafah où la dynamique du trait de côte reste globalement stable sur la période étudiée.

\section{References:}

1. Barry M., (2016). Erosion côtière et impacts dans la commune de Kafountine (Basse Casamance). Mémoire de Master, UAS/Z, 121 p.

2. Boak, E.H., Turner, I.L., (2005). Shoreline Definition and Detection: A Review. Journal of Coastal Research, 21 (4), 688-703. West Palm Beach (Florida), ISSN 0749-0208.

3. Byrnes M., (1994). Shoreline position and nearshore bathymetric change, US Army Corps of Engineers. Waterways Experiment Station. VCSBURG. Report, CERC-94-9, p.61-143.

4. Crowell M., Leatherman S., Buckley M.K., (1993). Shoreline change rate analysis: long term versus short term data. Shore and Beach, vol. $61, \mathrm{n}^{\circ} 2$, p. $13-20$.

5. Diop S., (1900) La Côte ouest-africaine, du Saloum (Sénégal) à la Mellacorée (République de Guinée). Paris : ORSTOM, 1990, 366 p.

6. Djagouna ERIC M. Valere., (2016). Cartographie De La Dynamique Du Trait De Côte A Grand-Lahou : Utilisation De L'outil «Digital Shoreline doi:10.19044/esj.2016.v12n36p327, Analysis,System(Dsas) » URL:http://dx.doi.org/10.19044/esj.2016.v12n36p327.

7. Emmanuel B., (2017). Etude des dynamiques du trait de côte de la région Bretagne à différentes échelles spatio-temporelles. Géographie. Université de Bretagne Occidentale — Brest. Français. <NNT : 2017BRES0010>.

8. Faye I., (2010). Dynamique du trait de côte sur les littoraux sableux de la Mauritanie à la Guinée-Bissau (Afrique de l'ouest) : approche régionale et locale par photo-interprétation, traitement d'images et analyses de cartes anciennes. Thèse de l'université de Bretagne occidentale, $321 \mathrm{p}$.

9. Fletcher C.H., Rooney J.J., Barbee M., LIM S.C., and Richmond B., (2003) Mapping shoreline change using digital orthophotogrammetry on Maui, Hawaii. Journal of Coastal, Research, Special Issue n ${ }^{\circ} 38$, pp $106-124$.

10. Himmelstoss, E.A., (2009). 'DSAS 4.0 Installation Instructions and User Guide' in: Thieler, E.R., Himmelstoss, E.A., Zichichi, J.L., and Ergul, Ayhan. 2009 Digital Shoreline Analysis System (DSAS) version 4.0 An ArcGIS extension for calculating shoreline change: 
U.S. Geological Survey Open-File Report 2008-1278. Updated for version 4.3.

11. Juigner M., (2012). Cinématique d'un trait de côte sableux en Vendée entre 1920 et 2010, Méthode et analyse, Géolittomer CNRSUMR 6554 LETG, Université de Nantes, 13p.

12. Le Berre I., Henaff A., Devogele T., MascreT A., et Wenzel F., (2016). «SPOT5 : un outil pertinent pour le suivi du trait de côte?», Norois [En ligne], 196 | 2005/3, mis en ligne le 15 décembre 2008, consulté le 30 septembre 2016. URL : http://norois.revues.org/378; DOI : 10.4000/norois.378.

13. Mayer E. R., (2006). Géomorphologie : Principe, Méthodologie et Pratique, guérin éditeur, Itée, 2006 ISBN 2 - 7601-6290-7, Canada, $496 \mathrm{p}$.

14. Moore 1. J., (2000). Shoreline mapping techniques. Journal of Coastal Research, vol. $16, n^{\circ} 1, p .111-124$.

15. Niang S., (2017). Dégradation chimique et mécanique des terres agricoles du Gandiolais (littoral nord du Sénégal), analyse des dynamiques actuelles d'adaptation, Thèse de Doctorat université Gaston berger de Saint Louis 355p.

16. Pennober G., 1999 : Analyse spatiale de l'environnement Côtier de l'Archipel des Bijagos (Guinée Bissau), Thèse de Doctorat, Université de Bretagne Occidentale, $232 \mathrm{p}$.

17. Robin M., Gourmelon F., (2016). «La télédétection et les SIG dans les espaces côtiers. Eléments de synthèse à travers le parcours de François Cuq », Norois [En ligne], 196 | 2005/3, mis en ligne le 15 décembre 2008, consulté le 12 août 2016. URL : http://norois.revues.org/368 ; DOI : $10.4000 /$ norois. 368 .

18. Ruë. O., (2005). Évolution du régime des vents des Côtes en Afrique de l'Ouest, Parc National du Banc d'Arguin.

19. Thieler, E. R., Martin, D., AND Ergul, A., (2003). The Digital Shoreline Analysis System, version 2.0: Shoreline change measurement software extension for ArcView: USGS U.S. Geological Survey Open-File Report 03-076. 